\title{
Performance Analysis of an OFDMA Transmission System in a Multicell Environment
}

\author{
Sophie Gault, Walid Hachem, and Philippe Ciblat
}

\begin{abstract}
The paper deals with design and performance analysis of orthogonal frequency-division multiple-access (OFDMA)based downlink cellular wireless communications. Due to a high degree of user mobility, the base station is assumed to have only a statistical knowledge of the users' channels. Relying on the ergodic capacities connected to the user rates, a subcarrier and power allocation that minimizes the total transmitted power is proposed. The allocation strategy requires only the knowledge of the channel statistics and the rate requirements for all users. An extension and a performance analysis of this allocation algorithm in a multicell environment working with a frequency reuse factor equal to one is also conducted. A condition for the multicell network to be able to satisfy all rate requirements is derived.
\end{abstract}

Index Terms-Ergodic capacity, frequency-hopping (FH), multicell interference (MCI), orthogonal frequency-division multiple access (OFDMA), power and subcarrier allocation.

\section{INTRODUCTION}

$\mathbf{I}$ $\mathrm{N}$ WIRELESS multiuser communications, orthogonal frequency-division multiple access (OFDMA) is a technique that combines discrete multicarrier modulation with an FDMA based on the dynamic allocation of subcarriers to users. The advantages of OFDMA include the flexibility in subcarrier attribution, the absence of multiuser interference due to subcarrier orthogonality, and the simplicity of the receiver. An OFDM modulation associated with a frequency-hopping $(\mathrm{FH})$ multiple-access technique can be also viewed as a spread spectrum technique. Consequently, FH-OFDMA offers the advantage of averaging interference as in a CDMA system and thus enables us to construct a cellular network with a frequency reuse factor between the adjacent cells equal to one [1], [2]. Thanks to these advantages, OFDMA receives a great deal of attention as a candidate for future wireless communication standards. However, the problem of the optimum subcarrier and power attribution to users as well as the robustness of OFDMA to intercell interference in a multicell setting are not fully understood. This paper

Paper approved by R. Fantacci, the Editor for Wireless Networks and Systems of the IEEE Communications Society. Manuscript received July 23, 2005; revised October 3, 2005 and January 23, 2006. This paper was presented in part at SPAWC, Cannes, France, 2006.

S. Gault was with the Telecommunications Department, Supélec, 91192 Gif-sur-Yvette, France. She is now with Motorola Labs, 91193 Gif-Sur-Yvette Cedex, France (e-mail: sophie.gault@motorola.com).

W. Hachem is with the Telecommunications Department, Supélec, 91192 Gif-sur-Yvette, France (e-mail: w.hachem@ieee.org).

P. Ciblat is with the Département Communications et Electronique, Ecole Nationale Supérieure des Télécommunications (ENST), 75013 Paris, France (e-mail: philippe.ciblat@enst.fr).

Digital Object Identifier 10.1109/TCOMM.2007.894101 is a contribution toward solving these problems for downlink communications.

The power and subcarrier allocation problem for OFDMA has been addressed by a number of contributions, among which [3]-[9] can be cited. These contributions assume the transfer functions of all users channels as being known to the base station (BS). In a multicell setting, the authors of [10] and [11] propose solutions that involve coordination between BSs through the existence of a radio network controller that gathers all channels state information in order to solve globally the subcarrier allocation problem. In this paper, we assume that these transfer functions are not available at the BS due to a high degree of user mobility and, for computational complexity reasons, that BSs do not cooperate. Assuming that the channels are random and frequency-selective and that the BS has only a statistical knowledge of these channels, the general problem that we address is the following: given the data rates required by the users, the BS has to find the optimum number of subcarriers and power per user in such a way that the rate requirements of all users are satisfied and, at the same time, the total transmitted power is minimum. Due to the time and frequency diversity of the users' channels and to the nonavailability of the channels' transfer functions at the BS site, we consider that a relevant measure of the achievable rate between the BS and a user is the so-called ergodic capacity. In practice, through $\mathrm{FH}$, the signal sent to a given user within a data frame visits a large number of this user's channel states and benefits from an averaging effect over the corresponding gains. This justifies the use of ergodic capacity as a performance measure.

One advantage of minimizing the BS transmitted power is to mitigate the interference that disturbs the neighboring cells. Nevertheless, this so-called multicell interference (MCI) still represents a fundamental obstacle against a possible increase of the whole cell capacity. In the second part of this paper, we analyze thoroughly the impact of MCI over the system performance. For a BS of interest, a simple way to combat the MCI that comes from the neighboring cells is to increase its own transmitted power. Consequently, in turn, the neighboring BSs will also have to increase their powers. If the system is to work, this process should converge. This leads to the issue of the whole system stability. In order to be able to conduct our performance analysis under the presence of MCI, and, in particular, to derive a condition for the system stability, we begin by assuming that the number of users in a cell and the signal bandwidth both grow toward infinity in such a way that the total rate (or capacity) of the BS per channel use ${ }^{1}$ converges toward a constant. A param-

\footnotetext{
${ }^{1}$ The capacity per channel use is the capacity divided by the channel bandwidth.
} 
eter of prime importance will emerge from our analysis: this is the mean rate per channel use and per cell volume unit required by the users in a cell. By considering an idealized network where all cells are identical and regularly spaced, we show that the network is stable if this rate is less than a given threshold. An analysis of this threshold in terms of certain system parameters like the cell radius and the power decay profile will also be conducted.

In Section II, we state the allocation problem for the singlecell case. Section III is devoted to the solution of the power and subcarrier optimization problem, which proves to be solvable by means of a Lagrangian formulation. The asymptotic regime in the number of users introduced above is described rigorously in Section IV. Under this regime, the issues of performance in presence of MCI and network stability are addressed in Section V. Finally, Section VI is devoted to the numerical illustrations of the results. We also compare the multicell OFDMA approach with an OFDMA technique assigning different sets of subcarriers to adjacent cells and thus working with a frequency reuse factor less than one.

In the paper, $\mathbb{E}[$.$] will denote the expectation operator.$ The (multivariate) complex-valued circular Gaussian distribution with mean a and covariance matrix $\boldsymbol{\Sigma}$ will be denoted $\mathcal{C N}(\mathbf{a}, \mathbf{\Sigma})$.

\section{Single-Cell Model}

We consider a downlink transmission where a BS serves $K$ users. The users channels are time-varying frequency-selective channels. The transmitted signal is parsed into frames, each corresponding to an orthogonal frequency-division multiplexing (OFDM) symbol of duration $T$ seconds. The channel impulse response of user $k$, which is assumed to be invariant during OFDM symbol $m$, is represented during this symbol by the vector $\mathbf{h}_{k}(m)=\left[h_{k}(m, 0), \ldots h_{k}(m, L-1)\right]^{\mathrm{T}}$, where $L$ is an upper bound on the user's channel lengths. Denoting by $N$ the number of subcarriers in an OFDM symbol (equivalently the number of channel uses per OFDM symbol), let $\mathbf{H}_{k}(m)=\left[H_{k}(m, 0), \ldots, H_{k}(m, N-1)\right]^{\mathrm{T}}$ be the vector that represents the transfer function of the channel of user $k$ at the $N$ Fourier frequencies of OFDM symbol $m$. In other words, $\mathbf{H}_{k}(m)=\sqrt{N} \mathbf{F}_{N, L} \mathbf{h}_{k}(m)$, where $\mathbf{F}_{N, L}$ is the $N \times L$ Fourier matrix in which the $(n, l)$ entry is given by $[\mathbf{F}]_{n, l}=(1 / \sqrt{N}) \exp (-2 i \pi n l / N)$ for $n=0, \ldots, N-1$ and $l=0, \ldots, L-1$. The signal $Y_{k}(m, n)$ received by user $k$ at subcarrier $n$ after the discrete fourier transformation of OFDM symbol $m$ is then written as

$$
Y_{k}(m, n)=H_{k}(m, n) S(m, n)+V_{k}(m, n)
$$

where $S(m, n)$ is the signal transmitted by the BS in the discrete Fourier domain and $V_{k}(m, n)$ is the additive noise received by user $k$ at subcarrier $n$ of OFDM symbol $m$. We assume that the two-dimensional (2-D) noise process $V_{k}(m, n)$ is white and that a sample of this process has the distribution $\mathcal{C N}\left(0, \sigma^{2}\right)$, where $\sigma^{2}$ refers to the noise variance. Recall that this variance is written as $\sigma^{2}=N_{0} B$, where $N_{0}$ is the noise power spectral density (PSD) and $B=N / T$ is the system bandwidth, or, equivalently, the number of channel uses per second. We formulate the following assumption regarding the users channels.

(A) The vector process $\mathbf{h}_{k}(m)$ is a random process with distribution $\mathcal{C N}\left(\mathbf{0}, \boldsymbol{\Sigma}_{k}\right)$, where

$$
\Sigma_{k}=\left[\begin{array}{ccc}
\varsigma_{k, 0}^{2} & & 0 \\
& \ddots & \\
0 & & \varsigma_{k, L-1}^{2}
\end{array}\right] .
$$

Assumption (A) states that the channel taps in the time domain are circular Gaussian and independent but they do not have necessarily the same variances. A consequence of (A) is that all entries of $\mathbf{H}_{k}(m)$, i.e., the transfer function coefficients in the discrete Fourier domain, have the distribution $\mathcal{C N}\left(0, \varsigma_{k}^{2}\right)$ with a variance $\varsigma_{k}^{2}=\sum_{l=0}^{L-1} \varsigma_{k, l}^{2}$. The fact that they have the same variance $\varsigma_{k}^{2}$ can be verified by inspecting the diagonal elements of the matrix $\mathbb{E}\left[\mathbf{H}_{k}(m) \mathbf{H}_{k}^{\mathrm{H}}(m)\right]=N \mathbf{F}_{N, L} \boldsymbol{\Sigma}_{k} \mathbf{F}_{N, L}^{\mathrm{H}}$. The result of this is that the gain-to-noise ratios (GNRs) $G_{k}(m, n)=$ $\left|H_{k}(m, n)\right|^{2} / N_{0}$ of user $k$ for $n \in\{0, \ldots, N-1\}$ and $m \in \mathbb{Z}$ are identically distributed.

In the sequel, it will be assumed that the receiver of user $k$ has the knowledge of its channel impulse response and of its noise power. Alternatively, at the BS, only the $K$ mean GNRs $a_{k}$ given by

$$
a_{k}=\mathbb{E}\left[G_{k}(m, n)\right]=\varsigma_{k}^{2} / N_{0}
$$

are assumed to be available. With these assumptions, we shall be interested all along this paper in the so-called ergodic Shannon capacities of these channels. Recall that the ergodic capacity can be approached by coding schemes that exploit properly the channel coherence bandwidth and/or its coherence time which we shall assume in the sequel.

In order to state our problem clearly, we begin by assuming that there is only one user $(K=1)$ communicating with the BS. To be able to reach the capacity, the transmitter has to send independent centered Gaussian signals over the $N$ subcarriers. In our case, since the random variables $G_{k}(m, n)$ are identically distributed, the capacity is reached when these Gaussian signals have the same variances. Assume that the user requires a rate of $\rho_{1}$ bits per channel use and denote by $E_{1}$ the minimum transmitted energy per channel use needed to satisfy this rate requirement. Then, $E_{1}$ satisfies $\rho_{1}=\mathbb{E}\left[\log \left(1+E_{1} G_{1}\right)\right]$, where $G_{1}$ is a random variable that has the same probability distribution as any of the random variables $G_{k}(m, n)$ and the expectation is taken with respect to this random variable. Note that the part of the energy devoted to the guard interval is neglected in this expression.

Let us turn now to the case where $K>1$. In this paper we restrict ourselves to a suboptimal user share strategy in the information-theoretic point of view. Indeed, we focus on the OFDMA scheme, which means that, for any subcarrier $n$ and OFDM symbol $m$, the signal $S(m, n)$ is allocated to a single user. We denote by $\gamma_{k}$ the sharing factor associated with user $k$. The factor $\gamma_{k}$ provides the proportion of time-frequency slots $(m, n)$ for which $S(m, n)$ is allocated to user $k$. By definition, we therefore have $\gamma_{k} \geq 0$ and $\sum_{k=1}^{K} \gamma_{k} \leq 1$. Once the sharing factors $\left\{\gamma_{1}, \ldots, \gamma_{K}\right\}$ are chosen, the practical allocation can be 
done in several ways: in theory, at one extreme, one can imagine that a user is given a whole OFDM symbol from time to time; at the other extreme, a user is given some fixed subset of the $N$ subcarriers of cardinality $n_{k}$ such that $n_{k} / N=\gamma_{k}$ up to a rounding error. In many practical situations, a more reasonable access scheme consists of allocating subcarriers to users according to some FH pattern [12]. Here, this pattern will be designed in such a way that constraints associated with the sharing factors $\gamma_{k}$ are respected.

Let $E_{k}=\mathbb{E}\left[|S(m, n)|^{2}\right] / B$ be the energy transmitted on the subcarrier $n$ of the OFDM symbol $m$ when the slot $(m, n)$ is destined to user $k$. The ergodic capacity per channel use $C_{k}$ given to user $k$ is then

$$
\begin{aligned}
C_{k} & =\gamma_{k} \mathbb{E}\left[\log \left(1+\frac{\left|H_{k}(m, n)\right|^{2} B E_{k}}{\sigma^{2}}\right)\right] \\
& =\gamma_{k} \mathbb{E}\left[\log \left(1+G_{k} E_{k}\right)\right]
\end{aligned}
$$

where $G_{k}$ is a random variable that has the same distribution as $G_{k}(m, n)$ and the expectation $\mathbb{E}$ is taken with respect to the distribution of $G_{k}$. Denoting by $Q_{k}$ the mean energy per channel use sent to user $k$, we have $Q_{k}=\gamma_{k} E_{k}$. The mean energy per channel use $Q$ transmitted by the BS is then

$$
Q=\sum_{k=1}^{K} Q_{k}
$$

Our problem is then the following: given a rate vector $\boldsymbol{\rho}=$ $\left[\rho_{1}, \ldots, \rho_{k}\right]^{\mathrm{T}}$, where $\rho_{k}$ is the capacity per channel use required by user $k$, find the energies $\left\{E_{k}\right\}$ and the sharing factors $\left\{\gamma_{k}\right\}$ such that the total transmitted energy $Q$ is minimum. Formally, this problem is written as follows: minimize $Q$ with the constraints

$$
\begin{aligned}
& -C_{k}+\rho_{k} \leq 0, \quad \text { for } k=1, \ldots, K \\
& \sum_{k=1}^{K} \gamma_{k}-1 \leq 0 .
\end{aligned}
$$

The capacity $C_{k}$ given by (3) is not a convex nor a concave function of $\left(\gamma_{k}, E_{k}\right)$. However, by writing

$$
C_{k}=\gamma_{k} \mathbb{E}\left[\log \left(1+G_{k} \frac{Q_{k}}{\gamma_{k}}\right)\right]
$$

it appears that $C_{k}$ is a concave function of $\left(\gamma_{k}, Q_{k}\right)$. Indeed, consider the function $f(x, y)=x \log (1+y / x)$ defined on $\mathbb{R}_{+}^{2}$. As the eigenvalues of the $2 \times 2$ Hessian matrix associated with $f(x, y)$ are 0 and $-\left(x^{2}+y^{2}\right) / x(x+y)^{2}$, this function is concave. It results that $C_{k}=\mathbb{E}\left[f\left(\gamma_{k}, G_{k} Q_{k}\right)\right]$ is concave.

In Section III, we will minimize the cost function (4) under the constraints (5) and (6) by using the Lagrangian multipliers.

\section{Allocation Algorithm}

Our constrained minimization problem (5) and (6) is convex in the vector parameter $\mathbf{x}=\left[\mathbf{q}^{\mathrm{T}}, \boldsymbol{\gamma}^{\mathrm{T}}\right]^{\mathrm{T}}$, where

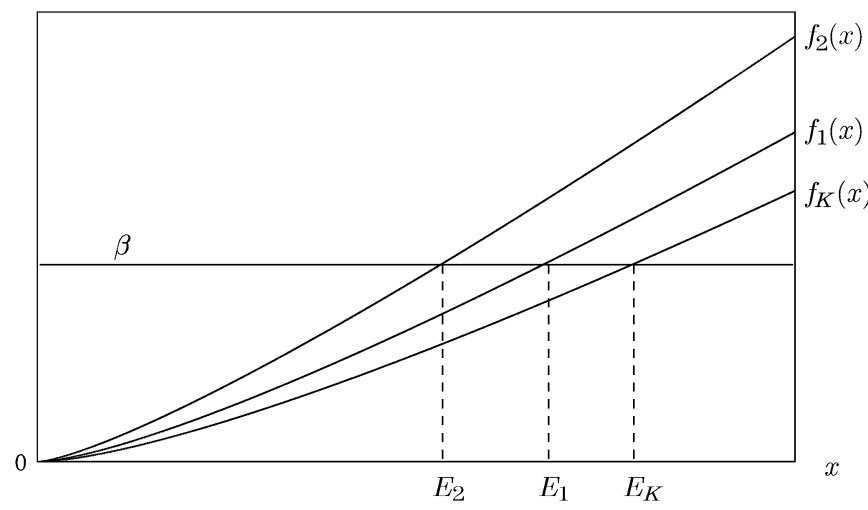

Fig. 1. Shapes of functions $f_{k}(x)$ and evolution of energies versus $\beta$.

$\mathbf{q}=\left[Q_{1}, \ldots, Q_{K}\right]^{\mathrm{T}}$ and $\boldsymbol{\gamma}=\left[\gamma_{1}, \ldots, \gamma_{K}\right]^{\mathrm{T}}$. The Lagrange/ Karush-Kuhn-Tucker (KKT) conditions are then written as

$$
\nabla_{\mathbf{x}} Q-\sum_{k=1}^{K} \lambda_{k} \nabla_{\mathbf{x}} C_{k}+\beta \nabla_{\mathbf{x}}\left(\sum_{k=1}^{K} \gamma_{k}\right)=0
$$

where $\nabla_{\mathbf{x}}$ denotes the gradient operator with respect to the vector $\mathbf{x}$, the positive real numbers $\lambda_{1}, \ldots, \lambda_{K}$ are the Lagrange multipliers associated with constraints (5), and the positive number $\beta$ is the Lagrange multiplier associated with the constraint (6). The multivariate (8) can be rewritten as the set of $2 K$ scalar equations $\lambda_{k} \partial C_{k} / \partial Q_{k}=1$ and $\lambda_{k} \partial C_{k} / \partial \gamma_{k}=\beta$ for $k=1, \ldots, K$. By developing the left-hand terms of these equations, we obtain

$$
\begin{array}{r}
\lambda_{k} \mathbb{E}\left[\frac{G_{k}}{1+G_{k} E_{k}}\right]=1 \\
\lambda_{k} \mathbb{E}\left[\log \left(1+G_{k} E_{k}\right)-\frac{G_{k} E_{k}}{1+G_{k} E_{k}}\right]=\beta
\end{array}
$$

for $k=1, \ldots, K$. By plugging (9) into (10), we have

$$
f_{1}\left(E_{1}\right)=f_{2}\left(E_{2}\right)=\cdots=f_{K}\left(E_{K}\right)=\beta
$$

where

$$
f_{k}(x)=\frac{\mathbb{E}\left[\log \left(1+x G_{k}\right)-\frac{x G_{k}}{1+x G_{k}}\right]}{\mathbb{E}\left[\frac{G_{k}}{1+x G_{k}}\right]}=\frac{\mathbb{E}\left[\log \left(1+x G_{k}\right)\right]}{\mathbb{E}\left[\frac{G_{k}}{1+x G_{k}}\right]}-x .
$$

Let us inspect the middle member of this expression. For every $a>0$, the function $g_{a}(x)=\log (1+a x)-a x /(1+a x)$ increases from zero to infinity as $x$ increases from zero to infinity. Therefore, the numerator increases from zero to infinity with $x$. As the denominator decreases with $x$, the function $f_{k}(x)$ increases from zero to infinity over the interval $[0, \infty)$ as shown in Fig. 1. The allocation algorithm is the following: initialize $\beta$ to a value close to zero. Compute the energies $E_{k}$ by solving numerically (11). To obtain the rate $\rho_{k}$, user $k$ needs the sharing factor $\gamma_{k}(\beta)$ given by

$$
\gamma_{k}(\beta)=\frac{\rho_{k}}{\mathbb{E}\left[\log \left(1+G_{k} E_{k}(\beta)\right)\right]}
$$


where $E_{k}(\beta)$ are the solutions of (11). If $\beta$ is too small, the energies $E_{k}(\beta)$ will be too small also and we will have $\sum_{k=1}^{K} \gamma_{k}(\beta)>1$. Increase $\beta$ until $\sum_{k=1}^{K} \gamma_{k}(\beta)=1$ is satisfied.

Let us give the expressions of the allocated energies and sharing factors with respect to the GNRs $a_{k}$. Since the random variables $H_{k}(m, n)$ are circular Gaussian, $G_{k}$ has the exponential distribution with mean $a_{k}$. Let $X_{e}$ be a positive random variable with the probability density $e^{-t}$, and let $f(x)$ be the function defined on $[0, \infty)$ as

$$
\begin{aligned}
f(x) & =\frac{\mathbb{E}\left[\log \left(1+x X_{e}\right)\right]}{\mathbb{E}\left[\frac{X_{e}}{1+x X_{e}}\right]}-x=\frac{\int \log (1+x t) e^{-t} d t}{\int \frac{t}{1+x t} e^{-t} d t}-x \\
& =\frac{e^{1 / x} x^{2} \operatorname{Ei}(1 / x)}{x-e^{1 / x} \operatorname{Ei}(1 / x)}-x
\end{aligned}
$$

where Eiis the so-called exponential integral function, defined as $\operatorname{Ei}(x)=\int_{x}^{\infty}\left(e^{-t} / t\right) d t$ for $x>0$.

As $G_{k}$ is exponentially distributed with mean $a_{k}$, it has the same distribution as $a_{k} X_{e}$. Therefore, from (12), we have

$$
f_{k}(x)=\frac{1}{a_{k}} f\left(a_{k} x\right) .
$$

Powers attribution connected to (11) can then be written as

$$
E_{k}(\beta)=\frac{1}{a_{k}} f^{(-1)}\left(a_{k} \beta\right)
$$

where $f^{(-1)}$ defined in $[0, \infty)$ is the inverse of $f$ with respect to composition. Thanks to (14), (13) can be rewritten as follows:

$$
\gamma_{k}(\beta)=\frac{\rho_{k}}{F\left(a_{k} \beta\right)}
$$

where $F(x)$ is the function defined on $\mathbb{R}_{+}$by

$$
F(x)=\mathbb{E}\left[\log \left(1+X_{e} f^{(-1)}(x)\right)\right] .
$$

It can be shown that $F(x)$ increases from zero to infinity as $x$ increases from zero to infinity. Moreover, $F(x)$ is continuous on $\mathbb{R}_{+}^{*}$.

Since $\sum_{k=1}^{K} \gamma_{k}(\beta)=1$, the multiplier $\beta$ is the unique solution to the following equation:

$$
\sum_{k=1}^{K} \frac{\rho_{k}}{F\left(a_{k} \beta\right)}=1 .
$$

It will also be useful to give the expression of $Q$, which is written as

$$
Q=\sum_{k=1}^{K} \frac{\rho_{k}}{a_{k}} \frac{1}{F\left(a_{k} \beta\right)} f^{(-1)}\left(a_{k} \beta\right) .
$$

We obtained an implicit closed-form expression for the minimal energy per channel use that enables us to ensure a rate $\rho_{k}$ for user $k$ in a single-cell environment.

\section{ASYMPTOTIC ANALYSIS}

The purpose of this section is to give an asymptotic expression of the transmitted energy per channel use of (16) and (17) in the asymptotic regime where the number of users $K$ in the cell grows toward infinity. Our aim is to obtain more tractable expressions that will be useful in particular in the multicell situation described in Section V. Assume that user $k$ requires a rate of $R_{k}$ nats per second. As the number of users grows to infinity, the total required rate $R^{(K)}=\sum_{k=1}^{K} R_{k}$ grows to infinity. In order to accommodate all of the users, we shall assume that the bandwidth $B$ also grows to infinity. The asymptotic regime will therefore be characterized by the fact that $K \rightarrow \infty, B \rightarrow \infty$, and $K / B \rightarrow \alpha$, where $\alpha$ is a positive constant. Note that, in this regime, the capacities per channel use (i.e., the spectral efficiencies) of the different users $\rho_{k}=R_{k} / B$ go to zero.

In order to ensure the convergence of the transmitted energy per channel use in the asymptotic regime and to obtain asymptotic expressions that can be interpreted simply, some additional hypotheses are required. The cell can be identified with a compact $\mathcal{C}$ included in $\mathbb{R}$ or in $\mathbb{R}^{2}$ according to whether the cell is one- or two-dimensional. It is frequent to model the GNR $a_{k}$ as being directly related to the location $x_{k}$ of mobile $k$. Here, $x_{k}$ is a one- or two-dimensional variable that represents a point of $\mathcal{C}$ in a coordinate system which origin is occupied by the BS. Getting back to (2), the variance $\varsigma_{k}^{2}$ will be written as $\varsigma_{k}^{2}=q\left(x_{k}\right)$ where $q(x)$ is a continuous function from $\mathcal{C}$ to $\mathbb{R}_{+}^{*}$ used to model the so-called path loss. With this model, we have $a_{k}=\pi\left(x_{k}\right)$, where $\pi(x)$ is the GNR profile defined as $\pi(x)=q(x) / N_{0}$. One widely used example for $q(x)$ is $q(x)=|x|^{-s}$, where $|x|$ denotes the distance between the mobile and the BS, and $s$ is a positive parameter that characterizes the rate of decrease of the signal power with distance. Remember that $q(x)$ is assumed to be defined on $\mathcal{C}$. Therefore, if $q(x)=|x|^{-s}$ is considered, the origin has to be excluded from $\mathcal{C}$. In this situation, it is often assumed that $\mathcal{C}=[-D,-d] \cup[d, D]$ in the one-dimensional (1-D) case, and $\mathcal{C}$ is the closed annulus delimited by circles with radii $d$ and $D$, where $d$ and $D$ are two real numbers such that $0<d<D$.

The two parameters of user $k$ required to implement the allocation algorithm (14)-(16) are $\rho_{k}=R_{k} / B$ and $a_{k}=q\left(x_{k}\right)$. By consequence, the user configuration can be equivalently characterized by the set of couples $\left\{\left(R_{k}, x_{k}\right)\right\}_{k=1, \ldots, K}$. Describing the set of parameters $\left\{\left(R_{k}, x_{k}\right)\right\}_{k=1, \ldots, K}$ is equivalent to providing the following positive measure $\nu^{(K)}$ acting on the Borel sets of $\mathbb{R}_{+} \times \mathbb{R}_{+}$:

$$
\nu^{(K)}(u, x)=\frac{1}{K} \sum_{k=1}^{K} \delta_{R_{k}, x_{k}}(u, x)
$$

where $\delta_{R_{k}, x_{k}}$ is the Dirac measure at the point $\left(R_{k}, x_{k}\right)$. It is realistic to assume that all required rates belong to an interval $\Delta_{R}=\left[R_{\min }, R_{\max }\right]$ of $\mathbb{R}_{+}^{*}$. By consequence, for every $K>0$, 


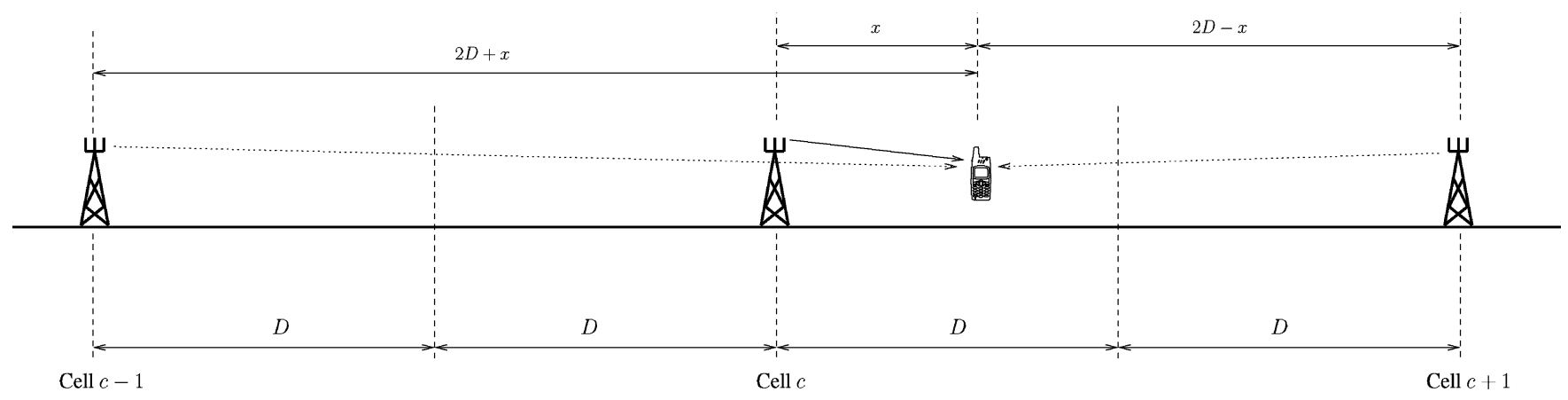

Fig. 2. Multicell environment.

the support of $\nu^{(K)}$ is included in the compact set $\Delta=\Delta_{R} \times \mathcal{C}$. Notice that $\nu^{(K)}$ is a positive measure satisfying $\int_{\Delta} d \nu^{(K)}=1$, and, as such, it is a probability measure. Using the fact that $\rho_{k}=$ $R_{k} / B$, (16) and (17) can be rewritten. respectively, as

$$
\frac{K}{B} \int_{\Delta} \frac{u}{F\left(\pi(x) \beta^{(K)}\right)} d \nu^{(K)}(u, x)=1
$$

and

$$
Q^{(K)}=\frac{K}{B} \int_{\Delta} \frac{u}{\pi(x)} \frac{f^{(-1)}\left(\pi(x) \beta^{(K)}\right)}{F\left(\pi(x) \beta^{(K)}\right)} d \nu^{(K)}(u, x) .
$$

In the last expression, the notation $Q^{(K)}$ is used instead of $Q$ to put ahead the fact that we now have a sequence of energies per channel use indexed by the number of users. The multiplier $\beta$ is denoted $\beta^{(K)}$ similarly. Convergence of this sequence will come from the following assumption:

(B1) As $K \rightarrow \infty$, the sequence of measures $\nu^{(K)}$ converges weakly to a probability measure $\nu$.

It is realistic to assume that the limit joint distribution $\nu$ of rates and users' locations is the measure product of a limit rate distribution times a limit location distribution. This is justified heuristically by a notion of independence between the rate requirements of the users and their locations.

(B2) The measure $\nu$ satisfies $d \nu(u, x)=d \zeta(u) \times d \lambda(x)$, where $\zeta$ is the limit distribution of rates and $\lambda$ is the limit distribution of the user locations $x_{k}$. Both $\zeta$ and $\lambda$ are probability measures. Here, $\times$ denotes the product of measures.

Typically, the measure $\lambda$ can be modeled as the uniform probability measure over $\mathcal{C}$; in other words, $d \lambda(x)=(1 /|\mathcal{C}|) d x$, where $|\mathcal{C}|$ is the cell volume. Concerning the limit rate distribution $\zeta$, denote by $\bar{R}=\int_{\Delta_{R}} u d \zeta(u)$ its mean. A parameter that will be of prime importance in the following is the mean rate $\bar{r}$ per channel use and per cell volume unit. It is given by $\bar{r}=\alpha \bar{R} /|\mathcal{C}|$ nats per channel use and per (squared) meter.

We turn now to the asymptotic expressions. We have the following theorem.

Theorem 1: Assume $K \rightarrow \infty$ in such a way that $K / B \rightarrow$ $\alpha>0$ and that the measure $\nu^{(K)}$ satisfies assumptions (B1) and (B2). Assume that $\pi(x)$ is continuous and satisfies $\pi(x)>0$ on $\mathcal{C}$. Then, $Q^{(K)}$ converges to $Q$ given by

$$
Q=\bar{r} \int_{\mathcal{C}} \frac{f^{(-1)}(\pi(x) \beta)}{\pi(x) F(\pi(x) \beta)}|\mathcal{C}| d \lambda(x)
$$

where $\beta$ is the unique positive number that satisfies

$$
\bar{r} \int_{\mathcal{C}} \frac{|\mathcal{C}|}{F(\pi(x) \beta)} d \lambda(x)=1 .
$$

This theorem, the proof of which is in Appendix A, is the main result of the paper in the single cell environment. One interesting consequence of Theorem 1 is that the rate distribution affects the asymptotic energy per channel use through its mean only. On other words, in the asymptotic regime, the minimal power consumed for achieving the individual user rates depends only on the global rate requirement. It does not depend on the particular form of the individual rate distribution.

\section{Multicell EnVIRONMENT}

In the GSM mobile cellular system, the spectrum is split into several subbands, and adjacent cells do not share the same subband. Consequently, there is no MCI coming from adjacent cells. However, such a system requires frequency planning and prevents soft handover. Therefore, advanced cellular systems (e.g., UMTS) will work with an universal frequency reuse to take benefit of the soft handover, of the macro-diversity, and of a flexible frequential management. To carry out a universal frequency reuse system, any spread-spectrum-based multiple-acces technique, such as DS-CDMA or FH-OFDMA, can be employed [13].

Consequently, in this section, we modify and analyze the behavior of the power allocation algorithm in a multicell environment, i.e., when, in addition to the background noise, the signal received by a user is corrupted by the signals sent by the BS of other cells. We prove that the power allocation strategy is quite different from the single-cell case and that there exists a maximum value for the rate. Beyond this threshold, the MCI strongly disturbs the transmission and does not enable us to get reliable transmission.

\section{A. Cell Model}

We consider for simplicity a 1-D cellular system that consists of a linear regular array of cells as shown in Fig. 2. In this figure, $D$ is half of the distance between two neighboring BSs, and a cell is included in the interval $[-D, D]$ if we identify its BS with the origin. Even if such a multicell model, studied in [14] and [15] is an ideal model, it provides some interesting guidelines that help to implement a practical cellular system. Here, to simplify our presentation, we furthermore assume that the MCI comes from the adjacent cells only; we thus neglect the 
MCI due to further cells. Suppose that, at OFDM symbol $m$, the signal sent by the BS on subcarrier $n$ is intended for user $k$. In a single-cell environment, the received signal $Y_{k}(m, n)$ is then written $Y_{k}(m, n)=H_{k}(m, n) S(m, n)+V_{k}(m, n)$ as shown in (1). To give the expression of the received signal in a multicell environment, let us number the cells as indicated in the figure and put the superscript ${ }^{(c)}$ to refer to the quantities located to cell number $c$. For instance, the signal transmitted by BS number $c$ will be denoted $S^{(c)}(m, n)$. Consistently with the notations of Section II, we denote by $\mathbf{h}_{k}^{\left(c^{\prime}, c\right)}(m)$ the impulse response of the channel that carries the signal of BS number $c^{\prime}$ to user $k$ of cell $c$ and by $\mathbf{H}_{k}^{\left(c^{\prime}, c\right)}(m)$ its discrete Fourier transform (DFT). With these notations, the signal received by user $k$ of cell $c$ at OFDM symbol $m$ and subcarrier $n$ becomes

$$
\begin{aligned}
Y_{k}^{(c)}(m, n)= & H_{k}^{(c)}(m, n) S^{(c)}(m, n) \\
& +H_{k}^{(c-1, c)}(m, n) S^{(c-1)}(m, n) \\
& +H_{k}^{(c+1, c)}(m, n) S^{(c+1)}(m, n) \\
& +V_{k}^{(c)}(m, n) .
\end{aligned}
$$

We assume that an FH algorithm is implemented in all cells and that this algorithm ensures that the signal of any user is equally distributed on all subcarriers. This results from this assumption that $\mathbb{E}\left[\left|S^{(c)}(m, n)\right|^{2}\right]=B Q^{(c)}$ for all $m$ and $n$. If we furthermore suppose that the intercell channels $\mathbf{h}_{k}^{\left(c^{\prime}, c\right)}(m)$ satisfy assumption (A), then the variance of $H_{k}^{(c-1, c)}(m, n)$ is independent of $m$ and $n$ as in Section II. In this case, the GNR $a_{k}^{(c)}$ of user $k$ in cell $c$ is written as shown at the bottom of the page. Let us consider the problem of the capacity derivation. The noise $V_{k}(m, n)$ in (1) is replaced in the multicell case by the noise $\tilde{V}_{k}(m, n)=$ $H_{k}^{(c-1, c)}(m, n) S^{(c-1)}(m, n)+H_{k}^{(c+1, c)}(m, n) S^{(c+1)}(m, n)+$ $V_{k}^{(c)}(m, n)$ as shown in (22). As this noise is clearly non-Gaussian, the capacity is in general difficult to derive. Nevertheless, if we endow $S^{(c)}(m, n)$ with the Gaussian distribution, then the associated mutual information between $S^{(c)}(m, n)$ and $Y_{k}^{(c)}(m, n)$ is a lower bound to the capacity. It is furthermore well known that when the variances of the information signal and the noise are fixed and the information signal is Gaussian, then the mutual information is minimum when the noise is Gaussian [16]. Therefore, we can easily derive a lower bound on the true capacity if we approximate the MCI noise by a Gaussian noise with the same variance. Then we are essentially led back to the situation of Section II.

As we shall require this lower bound to satisfy the rate constraints, the obtained power will represent an upper bound on the power necessary in theory to comply with these constraints.
In short, energies per channel use and shares are still computed according to (14)-(16) with the difference that $a_{k}$ is replaced by $a_{k}^{(c)}$. Note that the quantities $a_{k}^{(c)}$ can be consistently estimated by the BS number $c$.

Let us now consider the asymptotic regime in the multicell situation. In order to apply a path-loss model to the intercell channels, we extend the set of definition of the function $q(x)$ to $\mathcal{C} \cup(2 D+\mathcal{C}) \cup(2 D-\mathcal{C})$, where $2 D \pm \mathcal{C}=\{2 D \pm x, x \in \mathcal{C}\}$, and assume that $q(x)$ is continuous on this domain. With this extension, we have $\mathbb{E}\left[\left|H_{k}^{(c-1, c)}(m, n)\right|^{2}\right]=q(2 D+x)$ and $\mathbb{E}\left[\left|H_{k}^{(c+1, c)}(m, n)\right|^{2}\right]=q(2 D-x)$ (see Fig. 2). Let us denote by $\pi_{Q^{(c-1)}, Q^{(c+1)}}(x)$ the GNR profile in the conditions described by (22). This function is written as

$$
\begin{aligned}
\pi_{Q^{(c-1)}, Q^{(c+1)}} & (x) \\
& =\frac{q(x)}{Q^{(c-1)} q(2 D+x)+Q^{(c+1)} q(2 D-x)+N_{0}} .
\end{aligned}
$$

In a multicell setting, a lower bound on the total energy per channel use is therefore given by (20), where $\beta$ satisfies (21), and in both equations $\pi(x)$ is replaced by $\pi_{Q^{(c-1)}, Q^{(c+1)}}(x)$.

\section{B. Equilibrium Energy per Channel Use}

Any BS combats the MCI coming from its neighbors by increasing its own transmitted power. By doing so, however, it will increase the interference it produces with its neighboring cells, so that these cells in turn will have to increase their powers, and so forth. Here, we tackle the problem of finding a condition under which the whole cell array can nevertheless reach an equilibrium. Here we consider an infinite array and we assume that each cell satisfies the conditions of the asymptotic regime. To simplify our analysis, we assume that, at a certain moment that we call moment zero, all cells transmit at power $Q_{0} B$. For instance, one can imagine that moment zero is the moment where all BSs are "switched on" simultaneously, in which case one would have $Q_{0}=0$. After executing the allocation algorithm, each BS will transmit a signal with the energy per channel use given by (20) that we denote here as $Q_{1}$. At a later moment called moment one, the cells will execute again the algorithm simultaneously and then deliver the energy $Q_{2}$. By iterating, the energy $Q_{n+1}$ delivered by each BS at moment $n$ will be given by the following expressions. Let $\beta(Q, \bar{r})$ be the unique solution to the equation [see (18) and (19)]

$$
\bar{r} \int_{\mathcal{C}} \frac{|\mathcal{C}|}{F\left(\underline{\pi}_{Q}(x) \beta(Q, \bar{r})\right)} d \lambda(x)=1
$$

$$
a_{k}^{(c)}=\frac{\mathbb{E}\left[\left|H_{k}^{(c)}(m, n)\right|^{2}\right]}{Q^{(c-1)} \mathbb{E}\left[\left|H_{k}^{(c-1, c)}(m, n)\right|^{2}\right]+Q^{(c+1)} \mathbb{E}\left[\left|H_{k}^{(c+1, c)}(m, n)\right|^{2}\right]+N_{0}}
$$


where $\underline{\pi}_{Q}(x)$ is given by

$$
\underline{\pi}_{Q}(x)=\pi_{Q, Q}(x)=\frac{q(x)}{Q(q(2 D+x)+q(2 D-x))+N_{0}}
$$

and define $\xi(Q, \bar{r})$ as

$$
\xi(Q, \bar{r})=\bar{r} \int_{\mathcal{C}} \frac{f^{(-1)}\left(\underline{\pi}_{Q}(x) \beta(Q, \bar{r})\right)}{\pi_{Q}(x) F\left(\underline{\pi}_{Q}(x) \beta(Q, \bar{r})\right)}|\mathcal{C}| d \lambda(x)
$$

where $\xi(Q, \bar{r})$ is the total energy per channel use a cell needs to transmit to attain the mean rate of $\bar{r}$ nats per channel use and cell volume unit when its neighboring cells transmit at energy $Q$. The energy $Q_{n+1}$ will be given by

$$
Q_{n+1}=\xi\left(Q_{n}, \bar{r}\right)
$$

The convergence of this sequence is treated by the following theorem which is the main result of this section.

Theorem 2: Let $t(x)$ be defined in $\mathcal{C}$ as

$$
t(x)=\frac{q(x)}{q(2 D-x)+q(2 D+x)} .
$$

Assume that $t(x)$ is continuous and satisfies $t(x)>0$ in $\mathcal{C}$. Define $\psi(r)$ in $\mathbb{R}_{+}^{*}$ as

$$
\psi(r)=r \int_{\mathcal{C}} \frac{f^{(-1)}(t(x) b(r))}{t(x) F(t(x) b(r))}|\mathcal{C}| d \lambda(x)
$$

where $b(r)$ is the unique positive number that satisfies

$$
r \int_{\mathcal{C}} \frac{|\mathcal{C}|}{F(t(x) b(r))} d \lambda(x)=1
$$

Then, we have the following.

1) Equation $\psi(r)=1$ admits a unique solution $r_{0}>0$.

2) For any initial value $Q_{0} \geq 0$, if $\bar{r}<r_{0}$, then the sequence $\left(Q_{n}\right)$, the elements of which are given by (26), converges, and if $\bar{r} \geq r_{0}$, then it grows to infinity.

This theorem can be proven thanks to the following three lemmas.

Lemma 1: For every $r>0$, the function $\xi(Q, r)$ defined in (25) satisfies the following properties: $\xi(0, r)>0 ; \xi(Q, r)$ is increasing in the variable $Q$ on $\mathbb{R}_{+} ;$and $\xi(Q, r) / Q$ is decreasing in $Q$ on $\mathbb{R}_{+}^{*}$. (27).

Lemma 2: $\lim _{Q \rightarrow \infty} \xi(Q, r) / Q=\psi(r)$, where $\psi$ is given by

Lemma 3: $\lim _{r \rightarrow 0} \psi(r)=0$ and $\lim _{r \rightarrow \infty} \psi(r)=\infty$. Furthermore, $r \mapsto \psi(r)$ is increasing.

These lemmas are respectively proven in Appendices B-D. Finally, the proof of Theorem 1 is drawn in Appendix E.

Practically, this theorem indicates the following.

- If the rate is less than a certain threshold $r_{0}$, then the multicell system can operate.

- For a given achievable rate, i.e., a rate less than the threshold $r_{0}$, the proposed allocation strategy converges

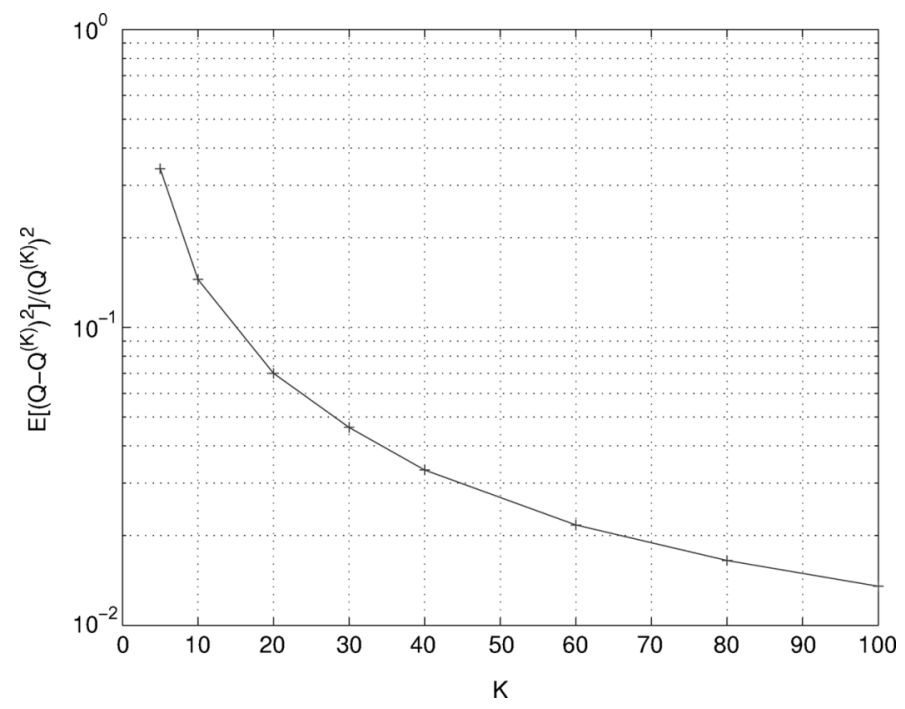

Fig. 3. Single-cell context: normalized MSE between the total power $Q$ required by the allocation algorithm and the power required in asymptotic regime $Q^{(K)}$ versus the number of users $K$ (FSL model).

and minimizes the power consumption. Notice that this part of the theorem is similar to the single-cell case.

\section{NUMERICAL ILLUSTRATIONS}

We begin by introducing the channel models that we considered. Simulations are carried out using three different path loss exponent values: $s=2,3$, and 3.5, as introduced in Section IV. We consider a free space loss (FSL) model characterized by a path-loss exponent $s=2$ and the so-called Okumura-Hata $(\mathrm{O}-\mathrm{H})$ model for open areas, which is widely used for predicting path loss in mobile wireless systems [17]. For the O-H case, we consider the cases $s=3$ and $s=3.5$.

The carrier frequency is $f_{0}=1.8 \mathrm{GHz}$. At this frequency, the basic equations for path loss in decibels are

$$
\begin{array}{ccc}
\text { FSL } & : & \pi_{\mathrm{dB}}^{\mathrm{I}}\left(x_{\mathrm{km}}\right)=20 \log _{10}\left(x_{\mathrm{km}}\right)+97.5 \\
\mathrm{O}-\mathrm{H} \quad s=3 & : & \pi_{\mathrm{dB}}^{\mathrm{II}}\left(x_{\mathrm{km}}\right)=30 \log _{10}\left(x_{\mathrm{km}}\right)+93.3 \\
\mathrm{O}-\mathrm{H} \quad s=3.5 & : & \pi_{\mathrm{dB}}^{\mathrm{III}}\left(x_{\mathrm{km}}\right)=35 \log _{10}\left(x_{\mathrm{km}}\right)+103.8
\end{array}
$$

where $x_{\mathrm{km}}$ is the distance in kilometers between the BS and the receiver. The default values for the cell inner radius $d$ and outer radius $D$ are set to $150 \mathrm{~m}$ and $5 \mathrm{~km}$, respectively. Finally, the signal bandwidth is $B=5 \mathrm{MHz}$ and the noise PSD is $N_{0}=$ $-170 \mathrm{dBm} / \mathrm{Hz}$.

We first validate the asymptotic analysis in a single-cell context with the FSL model. We consider a mean rate request $\bar{r}$ of 0.5 bit per second per channel use and per kilometer and compare the power $Q B$ required by our allocation algorithm [see (17)] for a number of users $K$ varying between 5 and 100 , and the power $Q^{(K)} B$ required in an asymptotic regime [see (20)]. In Fig. 3, we plotted the normalized MSE, i.e., $\left(Q-Q^{(K)}\right)^{2} /\left(Q^{(K)}\right)^{2}$.

In Figs. 4 and 5, we also compute the power required by the $\mathrm{BS}$ to reach a mean rate $\bar{r}$ of 0.2 and $0.5 \mathrm{~b} / \mathrm{s}$ per channel use and per kilometer, versus the cell radius for various channel models in the single cell context. We use the asymptotic approximations 


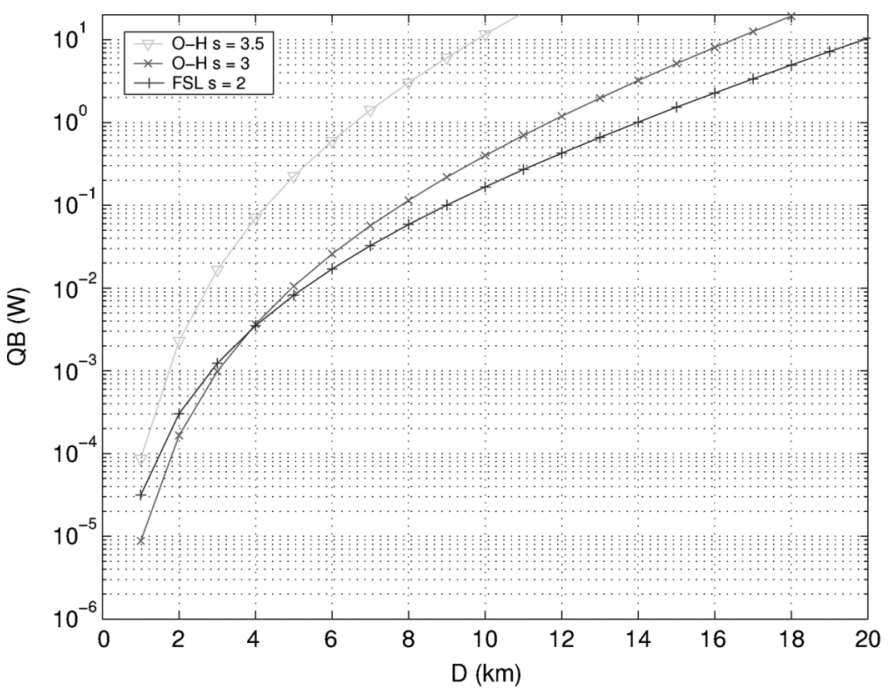

Fig. 4. Single-cell context: required power versus cell radius $D, \bar{r}=$ $0.2 \mathrm{~b} / \mathrm{s} / \mathrm{Hz} / \mathrm{km}$.

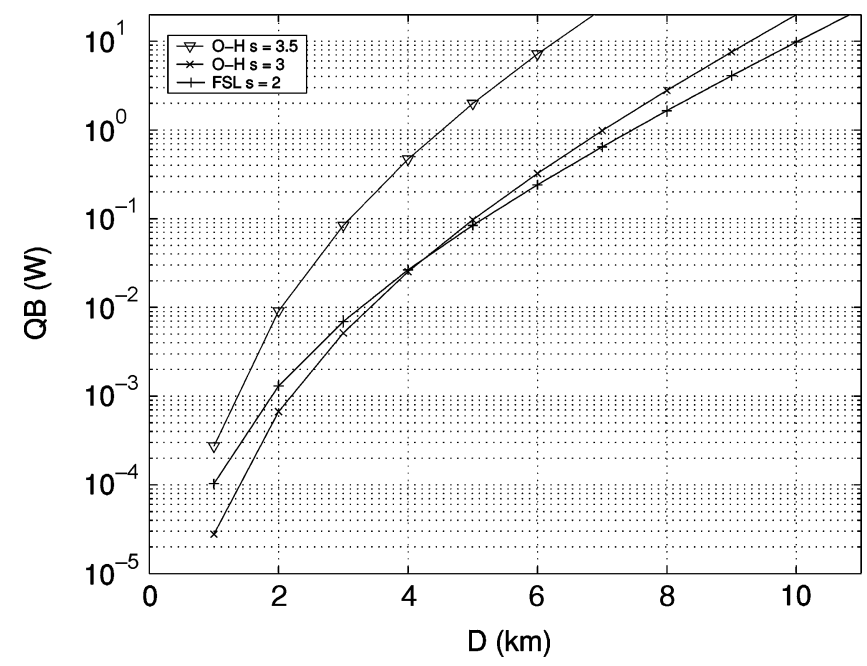

Fig. 5. Single-cell context: required power versus cell radius $D, \bar{r}=$ $0.5 \mathrm{~b} / \mathrm{s} / \mathrm{Hz} / \mathrm{km}$.

provided by (20) and (21). We assume that the transmitted power is limited to $20 \mathrm{~W}$, which corresponds to the upper border of the figures.

These curves give useful guidelines for cell dimensioning: given a constraint on the transmitted power, we directly deduce the corresponding size of the cell that can be covered by the BS. For instance, if the maximal transmitted power is $1 \mathrm{~W}$, the cell radius cannot be greater than $14 \mathrm{~km}$ for a mean rate requirement of $0.2 \mathrm{~b} / \mathrm{s} / \mathrm{Hz} / \mathrm{km}$ under the FSL model. Under the same conditions, the maximal radius becomes $7.5 \mathrm{~km}$ for a mean rate value of $0.5 \mathrm{~b} / \mathrm{s} / \mathrm{Hz} / \mathrm{km}$.

In a multicell environment, the BS coverage performance is seriously degraded by MCI. In Fig. 6, the mean required rate is set to $\bar{r}=0.2 \mathrm{~b} / \mathrm{s} / \mathrm{Hz} / \mathrm{km}$ as for Fig. 4. Fig. 6 shows that the maximal cell radius is reduced from 14 to $5.4 \mathrm{~km}$. Moreover, in Fig. 7, the maximal cell radius is shrinks from $7.5 \mathrm{~km}$ (see Fig. 5) to $2.2 \mathrm{~km}$ for a mean rate requirement of $0.5 \mathrm{~b} / \mathrm{s} / \mathrm{Hz} / \mathrm{km}$. Furthermore, it is worth noticing a major difference between single-cell

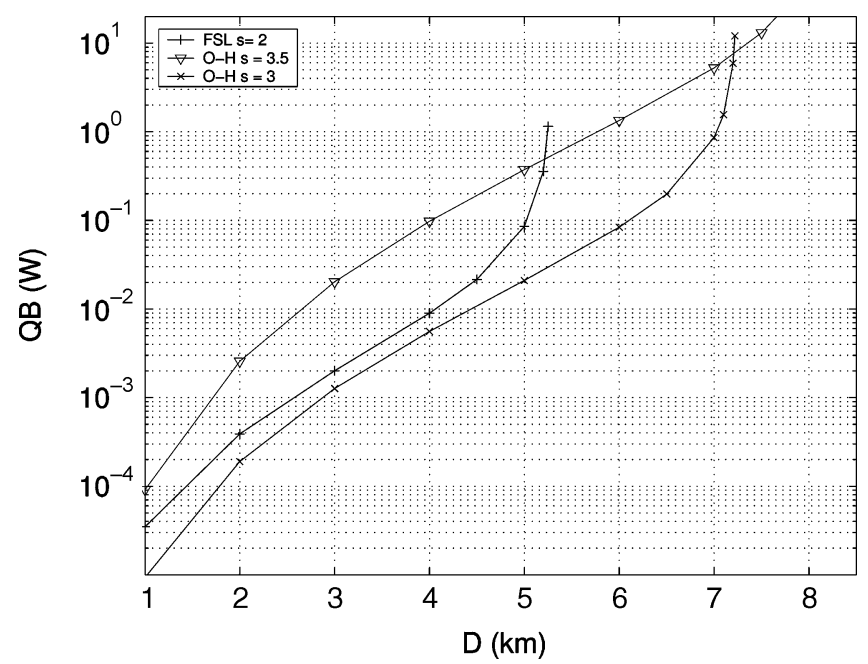

Fig. 6. Multicell context: required power versus cell radius $D, \bar{r}=$ $0.2 \mathrm{~b} / \mathrm{s} / \mathrm{Hz} / \mathrm{km}$.

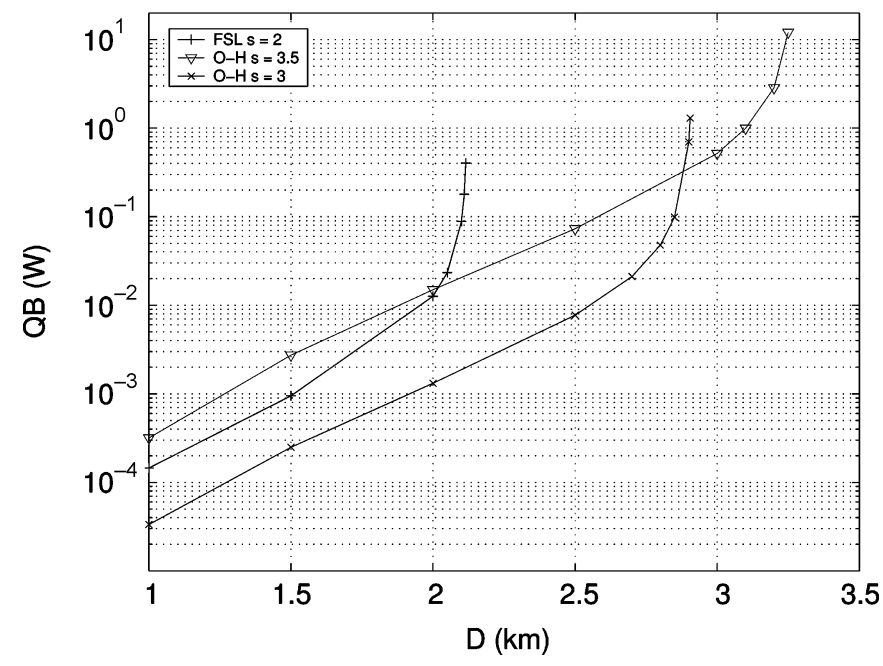

Fig. 7. Multicell context: required power versus cell radius $D, \bar{r}=$ $0.5 \mathrm{~b} / \mathrm{s} / \mathrm{Hz} / \mathrm{km}$.

and multicell contexts: the curves obtained in the multicell context grow to infinity when the cell radius $D$ reaches a certain threshold. This value depends on $s$ and on $\bar{r}$. For instance, in the free space model, for a mean rate requirement of $0.2 \mathrm{~b} / \mathrm{s} / \mathrm{Hz} / \mathrm{m}$, a limit is located at $D=5.4 \mathrm{~km}$.

To justify the existence of these limits, let us now focus on how the multicell results were obtained. Fig. 8 represents the function denoted $\xi(Q, \bar{r})$ given by (25) for three different values of $\bar{r}$. For each value of $Q$, we first find $\beta(Q, \bar{r})$ defined as the unique solution of (23), and then we compute $\xi(Q, \bar{r})$. The equilibrium power is given by the fixed point coordinates which can be determined geometrically by the intersection of $\xi(Q, \bar{r})$ with the first bisector, shown as a solid line in Fig. 8. The values of $Q$ corresponding to the different mean rate requirements are gathered in Table I. $Q$ naturally increases with $\bar{r}$, and so does $\psi(\bar{r})$. Therefore, as predicted by Theorem 2 , there exists a limit on the mean rate demand $r_{0}$, beyond which $\xi(Q, \bar{r})$ and the first bisector do not meet. Fig. 9 represents $r_{0}$ versus the cell radius in kilometers for the three path-loss models. For a given cell radius 
(a)

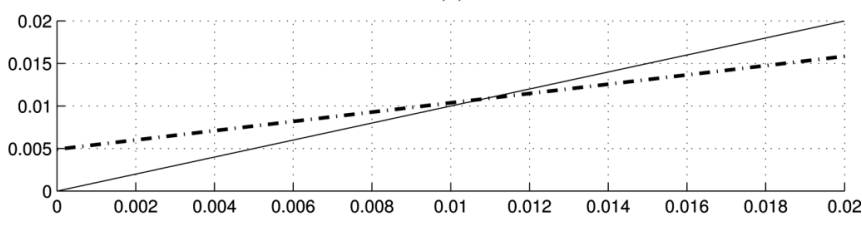

(b)
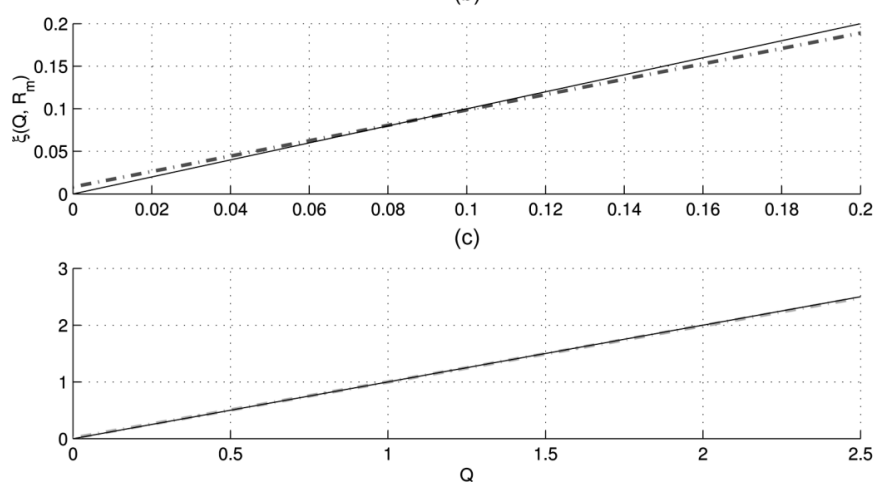

Fig. 8. Function $\xi(Q, \bar{r})$ versus $Q$ in a 5-km-radius cell for three different mean rate requirements: (a) $\bar{r}=0.15 \mathrm{~b} / \mathrm{s} / \mathrm{Hz} / \mathrm{km}$. (b) $\bar{r}=0.2 \mathrm{~b} / \mathrm{s} / \mathrm{Hz} / \mathrm{km}$. (c) $\bar{r}=$ $0.21 \mathrm{~b} / \mathrm{s} / \mathrm{Hz} / \mathrm{km}$ (FSL model).

TABLE I

EQUILIBRIUM POWER (INTERSECTION POINTS OF FIG. 8)

\begin{tabular}{|c|c|}
\hline $\bar{r}(\mathrm{bit} / \mathrm{s} / \mathrm{Hz} / \mathrm{km})$ & $Q B(\mathrm{~W})$ \\
\hline 0.15 & $1.08 \times 10^{-2}$ \\
\hline 0.2 & $8.55 \times 10^{-2}$ \\
\hline 0.21 & 0.95 \\
\hline
\end{tabular}

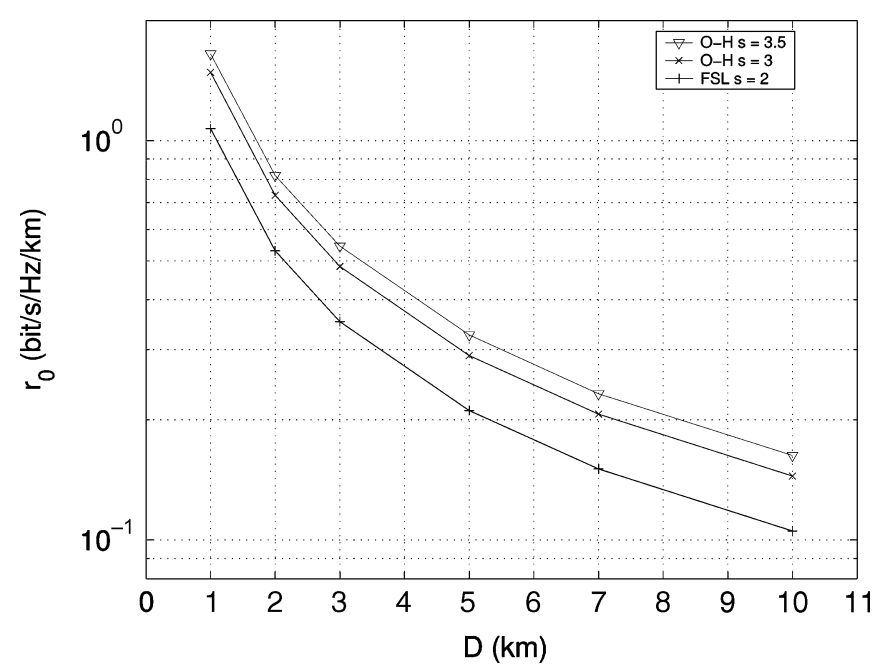

Fig. 9. Limit on the mean rate $R_{0}$ versus cell radius $D$.

$D$, any mean rate lower than $r_{0}$ can be satisfied i.e., the algorithm converges for any $\bar{r}<r_{0}$. Symmetrically, to any mean rate, $\bar{r}$ corresponds a maximal coverage radius $D$. As a consequence, for a given $\bar{r}$, when $D$ tends to the corresponding limit radius, $Q$ tends to infinity, which explains the presence of the asymptotes on Figs. 6 and 7.

Our FH-OFDMA allocation strategy is based on a universal frequency reuse (i.e., a frequency reuse factor of one). It would be interesting to compare this allocation strategy to

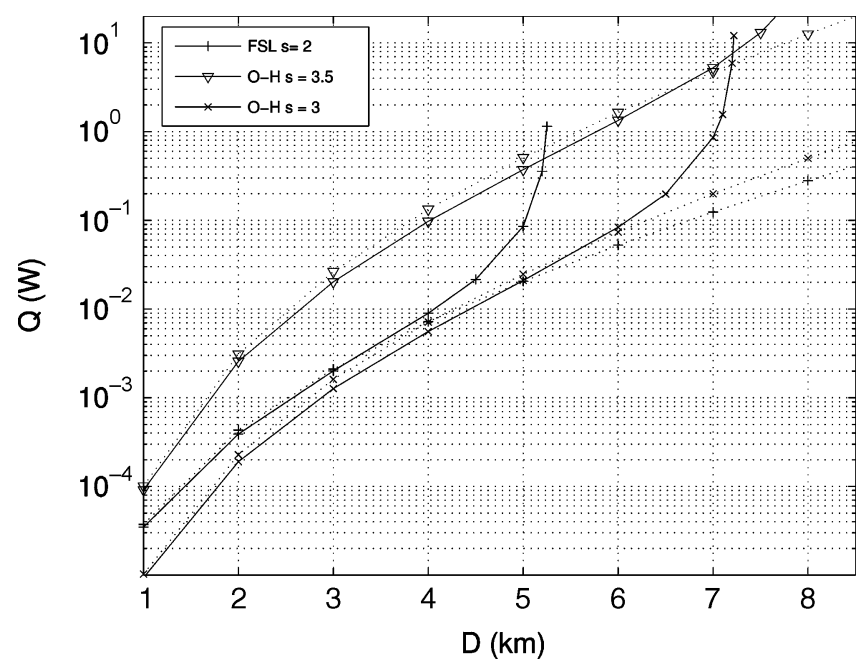

Fig. 10. Power per cell versus cell size with frequeucy reuse factor equal to 1 (plain) or $1 / 2$ (dashed).

a strategy that assigns different frequency bands to adjacent cells, which amounts to a frequency reuse factor of one half for one dimensional cells. With a frequency reuse factor of one, more (macro and frequency) diversity can be collected and the technical constraints due to frequency planning are avoided. In contrast, compared with the strategy with frequency planning, the signal-to-noise ratio per subcarrier degrades because of the MCI. In Fig. 10, we compare both strategies by plotting the consumed power versus the size of the cell for a given mean required rate per cell volume. In the case of FH-OFDMA with a reuse factor of one, we consider the mean rate $\bar{r}=0.2 \mathrm{~b} / \mathrm{s} / \mathrm{Hz} / \mathrm{km}$ and the power $Q B$ where $B=5 \mathrm{MHz}$ is the total bandwidth used by the system. In order to ensure the same mean rate requirement per cell volume, the approach with frequency planning is carried out by simply turning back to the single cell case (introduced in Section II) and by considering the mean rate requirement $\bar{r}^{\prime}=0.4 \mathrm{~b} / \mathrm{s} / \mathrm{Hz} / \mathrm{km}$ and a power equal to $Q^{\prime} B / 2$ where $Q^{\prime}$ is obtained via (16) and (17).

We notice that the required power per BS without frequency planning is slightly smaller than the power with frequency planning as long as the cell radius is smaller than a given threshold that depends on the path loss exponent $s$. Beyond this threshold, frequency planning is better from the point of vue of total consumed power. This threshold is equal to $3.2,5.6$, and $6.7 \mathrm{~km}$ for $s=2,3$, and 3.5 , respectively. For reasonable values of cell radius, it is useless to implement frequency planning.

\section{APPENDIX}

\section{A. Proof of Theorem 1}

Because $F(x)$ increases from zero to infinity on $\mathbb{R}_{+}$, it is clear that $\beta$ is the unique solution of (21). We shall show that $\beta^{(K)}$ given by (18) converges to $\beta$. Equation (21) can be rewritten as

$$
\alpha \int_{\Delta} \frac{u}{F(\pi(x) \beta)} d \nu(u, x)=1 .
$$

The function $F(x)$ is continuous and strictly positive on any compact subset of $\mathbb{R}_{+}^{*}$. Due to the assumptions on $\pi(x)$, the 
function $F(\pi(x) \beta)$ is continuous and satisfies $F(\pi(x) \beta)>0$ on the compact set $\Delta$ for every $\beta>0$. Therefore, for any $\beta>0$, $u / F(\pi(x) \beta)$ is continuous on $\Delta$. By applying standard results related to the convergence of measures [18], and by using (29), we therefore have

$$
\frac{K}{B} \int_{\Delta} \frac{u}{F(\pi(x) \beta)} d \nu^{(K)}(u, x) \rightarrow 1 .
$$

Let $\pi_{\min }=\min _{x \in \mathcal{C}} \pi(x)$ and $\pi_{\max }=\max _{x \in \mathcal{C}} \pi(x)$. By assumption, we have $\pi_{\min }>0$. From (18), we have the inequality

$$
\frac{K}{B} \frac{R_{\min }}{F\left(\beta^{(K)} \pi_{\max }\right)} \leq 1 \leq \frac{K}{B} \frac{R_{\max }}{F\left(\beta^{(K)} \pi_{\min }\right)} .
$$

Using the fact that $F(x)$ is increasing from zero to infinity, these inequalities show that, for sufficiently large $K$, all $\beta^{(K)}$ belong to a compact set $\left[\beta_{\min }, \beta_{\max }\right]$ with $\beta_{\min }>0$. By applying the same argument to (29), we show that $\beta \in\left[\beta_{\min }, \beta_{\max }\right]$ also. Thanks to (18), the convergence stated in (30) can be rewritten as

$$
\frac{K}{B} \int_{\Delta}\left(\frac{1}{F(\pi(x) \beta)}-\frac{1}{F\left(\pi(x) \beta^{(K)}\right)}\right) u d \nu^{(K)}(u, x) \rightarrow 0 .
$$

Assume that $\left|\beta^{(K)}-\beta\right|>\eta$ for some $\eta>0$. Then, $\mid \pi(x) \beta^{(K)}-$ $\pi(x) \beta \mid>\eta \pi_{\min }$ for all $x \in \mathcal{C}$. The function $F(x)$ is continuous on the compact interval $\left[\beta_{\min } \pi_{\min }, \beta_{\max } \pi_{\max }\right]$, hence $\left|F(\pi(x) \beta)-F\left(\pi(x) \beta^{(K)}\right)\right|$ is larger than a certain $\epsilon>0$ for all $x \in \mathcal{C}$. We shall therefore have

$$
\begin{aligned}
& \frac{K}{B}\left|\int_{\Delta}\left(\frac{1}{F(\pi(x) \beta)}-\frac{1}{F\left(\pi(x) \beta^{(K)}\right)}\right) u d \nu^{(K)}(u, x)\right| \\
& \quad=\frac{K}{B} \int_{\Delta} \frac{\left|F\left(\pi(x) \beta^{(K)}\right)-F(\pi(x) \beta)\right|}{F(\pi(x) \beta) F\left(\pi(x) \beta^{(K)}\right)} u d \nu^{(K)}(u, x) \\
& \quad \geq \frac{K}{B} \frac{\epsilon}{F\left(\beta_{\max } \pi_{\max }\right)^{2}} \int_{\Delta} u d \nu^{(K)}(u, x) \\
& \quad \geq \frac{K}{B} \frac{\epsilon}{F\left(\beta_{\max } \pi_{\max }\right)^{2}} R_{\min }
\end{aligned}
$$

which contradicts (31). Therefore, $\beta^{(K)} \rightarrow \beta$. With this, one can establish without difficulty the convergence of $Q^{(K)}$ toward $Q$ by considering (19) and (20).

\section{B. Proof of Lemma 1}

The first assertion can be established by noticing that $\xi(0, r)$ is the energy per channel use needed in the single-cell case to ensure a mean rate per channel use and cell volume unit equal to $r$.

To prove the second assertion, let us get back to the nonasymptotic regime and denote by $\mathbf{r}^{(K)}=\left[R_{1}, \ldots, R_{K}\right]$ a certain vector of $K$ rates, and by $\mathbf{x}^{(K)}=\left[x_{1}, \ldots, x_{K}\right]$ a vector of $K$ mobile locations. If the cell undergoes from its neighbors a MCI with power $Q B$, then the GNRs of the $K$ mobiles is given by $\underline{\pi}_{Q}\left(x_{k}\right)$. The total energy per channel use that the BS transmit after computing the allocation algorithm in these conditions is denoted by $\xi^{(K)}\left(\mathbf{r}^{(K)}, \mathbf{x}^{(K)}, Q\right)$. Associate with the vectors $\mathbf{r}^{(K)}$ and $\mathbf{x}^{(K)}$ the measure $\nu^{(K)}=1 K \sum_{k=1}^{K} \delta_{R_{k}, x_{k}}$ and assume that $\nu^{(K)}$ satisfies assumption (B1). Then, $\xi^{(K)}\left(\mathbf{r}^{(K)}, \mathbf{x}^{(K)}, Q\right) \rightarrow \xi(Q, r)$ as $K \rightarrow \infty$ thanks to Theorem 1. Therefore, if we prove that $\xi^{(K)}\left(\mathbf{r}^{(K)}, \mathbf{x}^{(K)}, Q\right)$ is increasing in the parameter $Q$ for all $K$, the second assertion will be proven thanks to the limit operator.

Let $g(x)$ be the function defined on $\mathbb{R}_{+}$as $g(x)=\mathbb{E}[\log (1+$ $\left.X_{e} x\right)$ ] where $X_{e}$ is a random variable with the exponential distribution with mean one. According to (7), the rate of user $k$ is provided by $R_{k}=B \gamma_{k} g\left(Q_{k} \underline{\pi}_{Q}\left(x_{k}\right) / \gamma_{k}\right)$ with the share $\gamma_{k}$ and the energy per channel use $Q_{k}$. Denote by $g^{(-1)}(x)$ the inverse on $\mathbb{R}_{+}$of $g(x)$ with respect to composition. Then, the energy $\xi^{(K)}\left(\mathbf{r}^{(K)}, \mathbf{x}^{(K)}, Q\right)$ is the minimum of the function

$$
\begin{aligned}
\Xi^{(K)}\left(\boldsymbol{\gamma}^{(K)}, \mathbf{r}^{(K)}, \mathbf{x}^{(K)}, Q\right) & =\sum_{k=1}^{K} Q_{k} \\
& =\sum_{k=1}^{K} \frac{\gamma_{k}}{\pi_{Q}\left(x_{k}\right)} g^{(-1)}\left(\frac{R_{k}}{\gamma_{k} B}\right)
\end{aligned}
$$

with respect to the vector $\boldsymbol{\gamma}^{(K)}=\left[\gamma_{1}, \ldots, \gamma_{K}\right]_{K}^{\mathrm{T}}$ over the unit simplex $\mathcal{S}=\left\{\gamma^{(K)}: \gamma_{1} \geq 0, \ldots, \gamma_{K} \geq 0, \sum_{k=1}^{K} \gamma_{k} \leq 1\right\}$. If $Q_{1} \geq Q_{2}$, then $\underline{\pi}_{Q_{1}}\left(x_{k}\right) \leq \underline{\pi}_{Q_{2}}\left(x_{k}\right)$ for all $k$, and therefore $\Xi^{(K)}\left(\boldsymbol{\gamma}^{(K)}, \mathbf{r}^{(K)}, \mathbf{x}^{(K)}, Q_{1}\right) \stackrel{\geq}{\geq} \Xi^{(K)}\left(\boldsymbol{\gamma}^{(K)}, \mathbf{r}^{(K)}, \mathbf{x}^{(K)}, Q_{2}\right)$ for every $\gamma^{(K)} \in \mathcal{S}$. Recall that, if two real functions $f_{1}$ and $f_{2}$ satisfy $f_{1}(x) \geq f_{2}(x)$ on some set $S$, then $\min _{x \in S} f_{1}(x) \geq \min _{x \in S} f_{2}(x)$. This implies that $\xi^{(K)}\left(\mathbf{r}^{(K)}, \mathbf{x}^{(K)}, Q_{1}\right) \geq \xi^{(K)}\left(\mathbf{r}^{(K)}, \mathbf{x}^{(K)}, Q_{2}\right)$, which proves the second assertion.

To prove the third assertion, we notice that

$$
\begin{aligned}
& \frac{\Xi^{(K)}\left(\boldsymbol{\gamma}^{(K)}, \mathbf{r}^{(K)}, \mathbf{x}^{(K)}, Q\right)}{Q} \\
& \quad=\sum_{k=1}^{K} \gamma_{k} \frac{q\left(2 D+x_{k}\right)+q\left(2 D-x_{k}\right)+\frac{\sigma^{2}}{Q}}{q\left(x_{k}\right)} g^{(-1)}\left(\frac{R_{k}}{\gamma_{k} B}\right)
\end{aligned}
$$

over $\mathcal{S}$. It is clear that, for $Q_{1} \geq Q_{2}$, $\Xi^{(K)}\left(\boldsymbol{\gamma}^{(K)}, \mathbf{r}^{(K)}, \mathbf{x}^{(K)}, Q_{1}\right) / Q_{1} \leq \Xi^{(K)}\left(\boldsymbol{\gamma}^{(K)}, \mathbf{r}^{(K)}, \mathbf{x}^{(K)}, Q_{2}\right) / Q_{2}$. The result follows from the same argument as above.

\section{Proof of Lemma 2}

Equation (25) can now be rewritten as

$$
\frac{\xi(Q, r)}{Q}=r \int_{\mathcal{C}} \frac{f^{(-1)}\left(\frac{\beta(Q, r)}{Q} z(Q, x)\right)}{z(Q, x) F\left(\frac{\beta(Q, r)}{Q} z(Q, x)\right)}|\mathcal{C}| d \lambda(x)
$$

where

$$
z(Q, x)=\frac{q(x)}{q(2 D-x)+q(2 D+x)+N_{0} / Q}
$$

and where $\beta(Q, r)$ is the solution of (23).

It is obvious that $z(Q, x)-t(x) \rightarrow 0$ as $Q \rightarrow \infty$. Moreover, for any $A>0$, due to the nonnullity and the continuity of $q(x)$ on the compact $\mathcal{C}$, one can check that $z(Q, x)>C_{A}$ with $C_{A}>$ 0 , regardless of $Q>A$. 
Now we shall prove that $\beta(Q, r) / Q$ is bounded. Indeed, as $z(Q, x)<t(x)$, we have

$$
\begin{aligned}
1 & =r \int_{\mathcal{C}} \frac{|\mathcal{C}|}{F\left(\frac{\beta(Q, r)}{Q} z(Q, x)\right)} d \lambda(x) \\
& >r \int_{\mathcal{C}} \frac{|\mathcal{C}|}{F\left(\frac{\beta(Q, r)}{Q} t(x)\right)} d \lambda(x) \\
& \geq r \frac{|\mathcal{C}|}{F\left(\frac{\beta(Q, r)}{Q} t_{\max }\right)}
\end{aligned}
$$

where $t_{\max }=\max _{x \in \mathcal{C}} t(x)$. Since $F($.$) is increasing from zero$ to infinity, previous inequalities show that $\beta(Q, r) / Q$ is lowerbounded. Furthermore, by using the lower bound of $z(Q, x)$, we obtain that

$$
1=r \int_{\mathcal{C}} \frac{|\mathcal{C}|}{F\left(\frac{\beta(Q, r)}{Q} z(Q, x)\right)} d \lambda(x)<r \frac{|\mathcal{C}|}{F\left(\frac{\beta(Q, r)}{Q} C_{A}\right)} .
$$

For the same reasons as above, $\beta(Q, r) / Q$ is upper-bounded. Consequently

$$
\frac{\beta(Q, r)}{Q} z(Q, x)-\frac{\beta(Q, r)}{Q} t(x) \underset{Q \rightarrow \infty}{\longrightarrow} 0 .
$$

As $x \mapsto(\beta(Q, r) / Q) z(Q, x)$ is strictly positive and continuous on the compact $\mathcal{C}$, and as $F($.$) is also strictly positive and contin-$ uous on $\mathbb{R}^{+}$, we get that $x \mapsto F((\beta(Q, r) / Q) z(Q, x))$ is strictly positive and continuous on the compact $\mathcal{C}$. Therefore, we obtain

$$
r \int_{\mathcal{C}} \frac{|\mathcal{C}|}{F\left(\frac{\beta(Q, r)}{Q} t(x)\right)} d \lambda(x) \underset{Q \rightarrow \infty}{\longrightarrow} 1 .
$$

Consequently due to the continuity of $F($.$) and the unicity of$ the solution of (23), we have that $\beta(Q, r) / Q$ converges toward $b(r)$ as $Q \rightarrow \infty$. Plugging in (32), we obtain the result.

\section{Proof of Lemma 3}

We begin by showing that $b(r)$ increases from zero to infinity on $\mathbb{R}_{+}$. It is clear by inspecting (28) that $b(r)$ is an increasing function. Let us show that $b(r) \rightarrow 0$ as $r \rightarrow 0$. Assume that $b(r)>\eta$ for a given $\eta>0$. Then, $F(b(r) t(x))>$ $F\left(\eta \min _{x \in \mathcal{C}}(t(x))\right)$ on $\mathcal{C}$ and therefore

$$
\int_{\mathcal{C}} \frac{|\mathcal{C}|}{F(b(r) t(x))} d \lambda(x)<\frac{|\mathcal{C}|}{F\left(\eta \min _{x \in \mathcal{C}}(t(x))\right)} .
$$

From (28), we then have $b(r)>\eta \Rightarrow r>C$ with $C=$ $F\left(\eta \min _{x \in \mathcal{C}}(t(x))\right) /|\mathcal{C}|>0$. This implies that $b(r) \rightarrow 0$ as $r \rightarrow 0$. One can show similarly that $b(r) \rightarrow \infty$ as $r \rightarrow \infty$.

The function $\psi(r)$ can be decomposed as follows:

$$
\psi(r)=|\mathcal{C}| r \int_{\mathcal{C}} \frac{\phi(g(r, x))}{t(x)} d \lambda(x)
$$

where

$$
\phi(\rho)=\frac{\rho}{\mathbb{E}_{X_{e}}\left[\log \left(1+X_{e} \rho\right)\right]}=\frac{\rho}{e^{1 / \rho} \operatorname{Ei}(1 / \rho)}
$$

and

$$
g(r, x)=f^{(-1)}(t(x) b(r)) .
$$

It is easy to check that $\rho \mapsto \phi(\rho)$ and $r \mapsto g(r, x)$ for each fixed $x$ are increasing functions. According to (35), we deduce that $\psi$ is increasing.

\section{E. Proof of Theorem 2}

From Lemmas 1 and $2, \xi(Q, r) / Q$ decreases from infinity to $\psi(r)$ and, by Lemma 3, $\psi(r)<1$ if and only if $r<r_{0}$. Consequently, the equation $\xi(Q, r)=Q$ admits a solution (which is unique) denoted by $Q_{s}$ if and only if $r<r_{0}$. In such a case, the sequence $\left(Q_{n}\right)$ converges to $Q_{s}$ regardless of the initial value of $Q_{0}$. Indeed, if $Q_{0}<Q_{s}$, then the sequence $\left(Q_{n}\right)$ is increasing and bounded: as $\xi(Q, r)>Q$ for $Q<Q_{s}$, we have $Q_{1}=\xi\left(Q_{0}, r\right) \geq Q_{0}$. Assume that $Q_{n} \geq Q_{n-1}$. Because $\xi(Q, r)$ is increasing in $Q$ as stated in lemma 1 , we have $Q_{n+1}=\xi\left(Q_{n}, r\right) \geq \xi\left(Q_{n-1}, r\right)=Q_{n}$. Therefore, $\left(Q_{n}\right)$ is increasing. As $Q_{0}<Q_{s}$ and $\xi(Q, r)$ is increasing, $Q_{1}=\xi\left(Q_{0}, r\right) \leq \xi\left(Q_{s}, r\right)=Q_{s}$, and, by the same argument, $Q_{n} \leq Q_{s}$ for every $n$. Therefore, $\left(Q_{n}\right)$ is increasing and bounded and thus converges. Since $Q \mapsto \xi(Q, r)$ is continuous and $Q_{s}$ is the unique solution of $\xi(Q, r)=Q$, the sequence $\left(Q_{n}\right)$ converges toward $Q_{s}$. By a similar argument, one shows that if $Q_{0}>Q_{s}$, then the sequence $\left(Q_{n}\right)$ decreases toward $Q_{s}$.

It remains to prove that, if $r \geq r_{0}$, then $\left(Q_{n}\right)$ diverges. Here, we have $\xi(Q, r)>Q$ for any value of $Q$. Therefore, the sequence $\left(Q_{n}\right)$ is increasing. Let us show that it is unbounded. Assume the contrary. In other words, there exists $\bar{Q}>0$ such that $Q_{n}<\bar{Q}$ for every $n$. Let $e=\xi(\bar{Q}, r) / \bar{Q}-1$. Because $r \geq r_{0}$, we have $e>0$. As $\xi(Q, r) / Q$ is decreasing, we have $\xi(Q, r) / Q-1 \geq e$ for every $Q<\bar{Q}$. By consequence, the elements of $\left(Q_{n}\right)$ satisfy $Q_{n+1}-Q_{n} \geq Q_{n} e \geq Q_{1} e$ for every $n$. Therefore, for $n \geq \bar{Q} / Q_{1} e$, we have $Q_{n}>\bar{Q}$ which is a contradiction.

\section{REFERENCES}

[1] K. Stamatiou and J. Proakis, "A performance analysis of coded frequency-Hopped OFDMA," in Proc. IEEE Wireless Commun. Networking Conf., 2005, pp. 1132-1137.

[2] R. Laroia, S. Uppala, and J. Li, "Designing a mobile broadband wireless access network," IEEE Signal Process. Mag., pp. 20-28, Sep. 2004.

[3] C. Y. Wong, R. S. Cheng, K. B. Letaief, and R. D. Murch, "Multiuser OFDM with adaptive subcarrier, bit and power allocation," IEEE J. Sel. Areas Commun., vol. 17, no. 10, pp. 1747-1758, Oct. 1999.

[4] D. Kivanc and H. Liu, "Subcarrier allocation and power control for OFDMA," in Proc. 34th Asilomar Conf. Signals, Syst. Comput., 2000, pp. $147-151$.

[5] S. Pietrzyk and G. J. M. Janssen, "Multiuser subcarrier allocation for QoS provision in the OFDMA systems," in Proc. IEEE Veh. Technol. Conf., 2002, pp. 1077-1081.

[6] M. Ergen, S. Coleri, and P. Varaiya, "QoS aware adaptive resource allocation techniques for fair scheduling in OFDMA based broadband wireless access systems," IEEE Trans. Broadcasting, vol. 49, no. 4, pp. 362-370, Dec. 2003.

[7] D. Kivanc, G. Li, and H. Liu, "Computationally efficient bandwidth allocation and power control for OFDMA," IEEE Trans. Wireless Commun., vol. 2, no. 6, pp. 1150-1158, Nov. 2003.

[8] J. Li, H. Kim, Y. Lee, and Y. Kim, "A novel broadband wireless OFDMA scheme for downlink in cellular communications," in Proc. IEEE Wireless Commun. Networking Conf., 2003, pp. 1907-1911. 
[9] G. Song and Y. Li, "Cross-layer optimization for OFDM wireless networks-Part I: Theoretical framework," IEEE Trans. Wireless Commun., vol. 4, no. 2, pp. 614-624, Mar. 2005.

[10] H. Kim, Y. Han, and J. Koo, "Optimal subchannel allocation scheme in multicell OFDMA systems," in Proc. IEEE Veh. Technol. Conf., 2004, pp. 1821-1825.

[11] Z. Han, F. R. Farrokhi, Z. Ji, and K. J. R. Liu, "Capacity optimization using subspace method over multicell OFDMA networks," in Proc. IEEE Wireless Commun. Networking Conf., 2004, pp. 2393-2398.

[12] R. Laroia, S. Uppala, and J. Li, "Designing a mobile broadband wireless access network," IEEE Signal Process. Mag., vol. 21, no. 5, pp. 20-28, Sep. 2004.

[13] D. Tse and P. Viswanath, Fundamentals of Wireless Communication. Cambridge, U.K.: Cambridge Univ. Press, 2005.

[14] A. D. Wyner, "Shannon-theoretic approach to a Gaussian cellular multiple-access channel," IEEE Trans. Inf. Theory, vol. 40, no. 6, pp. 1713-1727, Nov. 1994.

[15] B. M. Zaidel, S. Shamai, and S. Verdú, "Multicell uplink spectral efficiency of coded DS-CDMA with random signatures," IEEE J. Sel. Areas Commun., vol. 19, no. 8, pp. 1556-1568, Aug. 2001.

[16] T. Cover and J. Thomas, Elements of Information Theory. New York: Wiley, 1991.

[17] Digital Mobile Radio Towards Future Generation Systems, Final Report Tech. Rep., Eur. Communities, EUR 18957, 1999, COST Action 231.

[18] P. Billingsley, Probability and Measure, 3rd ed. New York: Wiley, 1995.

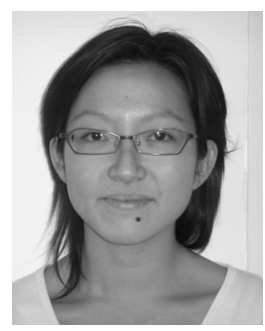

Sophie Gault was born in Rennes, France, in 1980. She received the Engineering degree and the M.Sc. degree from the Institut National des Sciences Appliquées (INSA), Rennes, France, in 2002, and the Ph.D. degree from the Université de of Paris Sud, Orsay, France, in 2005.

She has worked on last-mile access powerline communications with Supélec and Ecole Nationale Supérieure des Télécommunications (ENST) in the framework of the French RNRT project IDILE. Since October 2005, she has been with Motorola
Labs. Her present topics of interest include network design and resource allocation strategies for the future generation of broadband wireless access.

Walid Hachem was born in Bhamdoun, Lebanon, in 1967. He received the Engineering degree in telecommunications from St. Joseph University (ESIB), Beirut, Lebanon, in 1989, the M.S. degree in signal processing from Télécom Paris (ENST), Paris, France, in 1990, the Ph.D. degree in signal processing from Université de Marne-La-Vallée, Marne-La-Vallée, France, in 2000, and the Habilitation à Diriger des Recherches from Université de Paris-Sud, Orsay, France, in 2006.

He was with Philips T.R.T., where he was involved with telephone modems, and then joined FERMA, working on DSP processing for voice servers. He is currently an Associate Professor with the Ecole Supérieure d'Electricite (Supélec), Paris. His research interests concern the asymptotic analysis of multiuser systems based on the study of large random matrices, channel estimation and synchronization for multicarrier systems, and multiuser mobile communications.

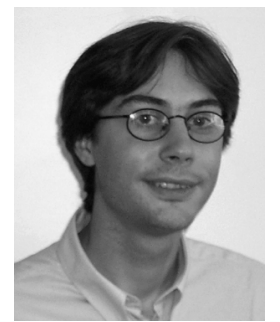

Philippe Ciblat was born in Paris, France, in 1973. $\mathrm{He}$ received the Engineering degree from the Ecole Nationale Supérieure des Télécommunications (ENST) and the DEA degree (French equivalent to the M.Sc. degree) in automatic control and signal processing from the Université de Paris-Sud, Orsay, France, both in 1996, and the Ph.D. degree from Laboratoire Système de Communication of the Université de Marne-la-Vallée, Marne-la-Vallée, France, in 2000. His Ph.D. dissertation focused on the estimation issue in noncooperative telecommunications. In 2001, he was a Postdoctoral Researcher with the Laboratoire des Télécommunications, Université Catholique de Louvain, Louvain, Belgium. At the end of 2001, he joined the Département de Communications et Electronique, ENST, Paris, France, as an Associate Professor. His research areas include statistical and digital signal processing (i.e., blind equalization, frequency estimation, and asymptotic performance analysis) and signal processing for digital communications (i.e., synchronization for OFDM modulations and the CDMA scheme, access technique and localization for UWB, and the design of global systems).

Dr. Ciblat has served as an Associate Editor for IEEE COMMUNICATIONS LETTERS since 2004. 